\title{
Padronização de metodologia de extração lipídica para análise isotópica de tecidos animais
}

\author{
Standardization of lipid extraction methodology for isotopic analysis of \\ animal tissues
}

\author{
Bruna Ewely da Silva Petinati ${ }^{1}$, Juliana Célia Denadai ${ }^{1}$, Robert Guaracy Aparecido Cardoso Araujo ${ }^{1^{*}}$, Rodrigo da Silva \\ Gennari $^{1}$, Thatiane Mendes Mitsunaga ${ }^{1}$, Evandro Tadeu Silva ${ }^{1}$, Maria Marcia Pereira Sartori ${ }^{2}$, Vladmir Eliodoro Costa ${ }^{3}$ \\ ${ }^{1}$ Faculdade de Medicina Veterinária e Zootecnia, Universidade do Estado de São Paulo (UNESP), Botucatu, SP, Brasil \\ ${ }^{2}$ Faculdade de Ciências Agronômicas, Universidade do Estado de São Paulo (UNESP), Botucatu, SP, Brasil \\ ${ }^{3}$ Instituto de Biociências, Universidade do Estado de São Paulo (UNESP), Botucatu, SP, Brasil
}

\section{Resumo}

Objetivou-se com este estudo determinar se os processos de extração lipídica alteram o valor isotópico no fígado e músculo de frangos, peixes, suínos e bovinos. Foram coletadas 24 amostras de fígado e músculo destes animais de interesse zootécnico, sendo divididas aleatoriamente em três tratamentos com oito repetições. As amostras foram coletadas com cuidado para que todas fossem do mesmo lote. Os tratamentos foram: desidratado - as amostras foram secadas durante 48 horas por estufa de ventilação forçada; Soxhlet - as amostras foram desengorduradas em éter etílico; e Folch - as amostras foram desengorduradas em clorofórmio/metanol. Os resultados isotópicos foram submetidos à análise multivariada discriminante linear por meio do pacote estatístico do software Minitab 16. A extração lipídica por éter interferiu nos valores isotópicos dos tecidos avaliados de frangos, suínos e pintado. A extração lipídica por clorofórmio/metanol interferiu nos valores isotópicos dos tecidos musculares de frangos e suínos. Não houve alterações nos valores isotópicos, independentemente do método de extração, para os tecidos de bovino e para tilápia do Nilo. Sugerese, portanto, a não extração lipídica em tecidos de animais com finalidade zootécnica, a fim de diminuir a interferência dos processos de extração lipídica nos valores isotópicos dos tecidos animais.

Palavras-chave: Carbono-13. Clorofórmio/metanol. Éter. Isótopos estáveis. Nitrogênio-15.

\section{Abstract}

Our aim was to determine if the isotopic value is altered by lipid extraction processes in the liver and muscle of chickens, fish, pigs, and cattle. We collected randomly twenty-four liver and muscle samples from these animals dividing them into three treatments with eight replicates each. Samples 
were carefully collected belonging to the same lot. The treatments were: dehydrated - dried for 48 hours by forced ventilation oven; Soxhlet - samples were degreased in ethyl ether; and Folch - samples were degreased in chloroform/ methanol. Isotopic results were submitted to linear discriminant multivariate analysis using the Minitab 16 statistical software package. Lipid extraction by ether interfered with the isotopic values of the evaluated chicken, pig and fish (Pseudoplatystoma corruscans) tissues. Lipid extraction by chloroform/methanol interfered in the isotopic values of the muscular tissues of chickens and pigs. There were no changes in isotopic values independent of the extraction method for bovine tissues and for tilapia fillet. It is suggested, therefore, the non-extraction of lipids from tissues of animals with zootechnical purpose in order to reduce the interference of the lipid extraction processes in the isotopic values of the animal tissues.

Keywords: Carbon-13. Chloroform/methanol. Ether. Nitrogen-15. Stable isotopes.

\section{Introdução}

A espectrometria de massas, por meio da análise da razão isotópica do carbono-13 $\left(\delta^{13} \mathrm{C}\right)$, tem sido usada com sucesso para testar a autenticidade e a qualidade de vários produtos, tais como sucos de frutas (Bricout e Koziet, 1987; Koziet et al., 1993), vinhos (Martin etal., 1988), mel (Brookes et al., 1991; White et al., 1998), produtos lácteos (Rossmann et al., 2000; Manca et al., 2001) e ovos (Denadai et al., 2011), além da caracterização e diferenciação do regime dietético de suínos Ibéricos, possibilitando a classificação dos animais de acordo com o tipo de alimentação recebida durante o período de engorda (González-Martin et al., 1999) e a certificação de origem e de qualidade de produtos de origem animal (Hargin, 1996; Monin, 1998; González-Martin et al., 2001). 0 enriquecimento relativo do $\delta^{13} \mathrm{C}$, associado ao do nitrogênio-15 $\left(\delta^{15} \mathrm{~N}\right)$, permitiu a certificação da origem geográfica e identificação do tipo de alimentação de ovinos (Piasentier et al., 2003).

Os estudos visam a complementação da técnica dos isótopos estáveis na rastreabilidade das farinhas de origem animal na alimentação das aves, detectável no produto final. Para o desenvolvimento dessa linha de pesquisa, entretanto, Oliveira (2005) evidenciou a necessidade de realização de estudos que contribuam para o conhecimento da assimilação isotópica do carbono em diferentes tecidos, em outras fases de criação das aves, e métodos que melhorem e tornem ágil o preparo das amostras, com a possibilidade de resultados mais rápidos.

Segundo Bury et al. (2008), em estudos com espécies ricas em lipídios, ao removê-los completamente, podem surgir problemas ao tentar obter valores confiáveis de $\delta^{13} \mathrm{C}$ e $\delta^{15} \mathrm{~N}$ através da análise de isótopos estáveis. A extração lipídica é realizada para normalizar a razão de isótopos estáveis de carbono, mas também tem sido demonstrado que afetam a razão de isótopos estáveis de nitrogênio em tecidos de peixes e invertebrados (Kiljunen et al., 2006; Sweeting et al., 2006; Post et al., 2007).

Atualmente os cientistas removem os lipídios dos tecidos e frações por diversos processos químicos, pois se sabe que os lipídios são desinrequecidos em $\delta^{13} \mathrm{C}$. Este processo, porém, vem sendo amplamente investigado e criticado devido à variação isotópica causada por cada um dos métodos. Além disso, as técnicas de extração de gordura são extensas, o que provoca lentidão na preparação das amostras. Para a melhoria da interpretação dos resultados isotópicos, fazem-se necessários estudos destes métodos e da interferência de cada um destes para posterior eleição da melhor técnica e padronização da metodologia de preparo das amostras no processo de rastreabilidade, pois desconfia-se que no processo de extração, além de ocorrer a retirada dos lipídeos, ocorra também a extração de moléculas empobrecidas em $\delta^{15} \mathrm{~N}$.

Portanto, a fim de padronizar o método a ser utilizado em experimentos futuros, o objetivo deste estudo foi determinar se os processos de extração de lipídeos alteram o valor isotópico do fígado e músculo de diversas espécies de interesse zootécnico.

\section{Material e métodos}

O experimento foi desenvolvido na Universidade Estadual Paulista (UNESP) de Botucatu, no Centro de Isótopos Estáveis Ambientais do Instituto de Biociências. As amostras de frangos foram 
adquiridas em supermercado, as de suínos em abatedouro experimental, as de peixes e de bovinos de experimento realizado na UNESP/Botucatu, com o cuidado de todas serem do mesmo lote para que a variável fosse apenas o processo de extração dos lipídios.

Foram avaliados os processos de desengorduramento do fígado e músculo de dois tecidos diferentes, um com alto percentual de gordura (APG) e outro com baixo percentual de gordura (BPG) de aves, peixes, suínos e bovinos. Os tecidos escolhidos para aves foram músculo peitoral (BPG) e músculo da coxa (APG). Dois tipos diferentes de peixes foram utilizados: tilápia (BPG) e pintado (APG). Os tecidos musculares escolhidos para suínos foram o masseter (BPG) e músculo semitendinoso (APG). E para tecido muscular de bovinos foram escolhidos lagarto (BPG) e acém (APG).

Foram coletadas 24 amostras de fígado e músculo destes animais, sendo divididas aleatoriamente em três tratamentos com oito repetições, onde os tratamentos foram constituídos de: desidratado secado durante 48 horas por estufa de ventilação forçada; Soxhlet-as amostras foram desengorduradas em éter etílico; e Folch - as amostras foram desengorduradas em clorofórmio/metanol.

\section{Descrição da análise isotópica}

As amostras desidratadas foram secadas em estufa de ventilação forçada (Marconi, modelo MA 035 , Piracicaba, Brasil) a $56^{\circ} \mathrm{C}$, por um período de 48 horas e, posteriormente, moídas criogenicamente (moinho criogênico modelo Spex - 6750 freezer/ mill, Metuchen, EUA) a $-196{ }^{\circ} \mathrm{C}$, método descrito por Carrijo et al. (2000) e Denadai et al. (2008). As amostras do Soxhlet foram secadas em estufa de ventilação forçada a $56{ }^{\circ} \mathrm{C}$, por um período de 48 horas, moídas criogenicamente e desengorduradas em éter etílico (Soxhlet), segundo Carrijo et al. (2000) e Denadai et al. (2008). No método de Folch, as amostras foram secadas em estufa de ventilação forçada a $56{ }^{\circ} \mathrm{C}$ por um período de 48 horas, moídas criogenicamente e desengorduradas em clorofórmio-metanol (2:1) (Folch et al., 1957).

A análise foi efetuada no Centro de Isótopos Estáveis do Instituto de Biociências da UNESP, Botucatu. Aproximadamente 50-70 $\mu$ g e 500-600 $\mu \mathrm{g}$ de amostras foram utilizadas para a medida das razões isotópicas de $\delta^{13} \mathrm{C} / \delta^{12} \mathrm{C}, \delta^{15} \mathrm{~N} / \delta^{14} \mathrm{~N}$, respectivamente, cujos gases $\mathrm{CO}_{2}$ e $\mathrm{N}_{2}$ são obtidos por combustão on-line com $\mathrm{o}$ espectrômetro de massa, os quais fornecem os valores do enriquecimento relativo de $\delta^{13} \mathrm{C}$ e $\delta^{15} \mathrm{~N}$ em relação ao seu respectivo padrão internacional.

Os valores da composição isotópica foram obtidos em relação aos padrões internacionais $\delta^{13} \mathrm{C}$ : V-PDB (Vienna Pee Dee Belemnite) e $\delta^{15} \mathrm{~N}: \mathrm{N}_{2}$ do ar atmosférico, com erro de análise da ordem de $0,2 \%$ e calculado pela equação:

$\delta \mathrm{X}($ amostra, padrão $)=[(\mathrm{R}$ amostra $/ \mathrm{R}$ padrão $)-1] \times 10^{3}$

$\delta \mathrm{X}$ representa o enriquecimento do isótopo menos abundante do elemento químico $\mathrm{X}\left(\delta^{13} \mathrm{C}\right.$ ou $\delta^{15} \mathrm{~N}$ ) da amostra em relação ao respectivo padrão internacional, e R representa a razão entre o isótopo menos abundante e o mais abundante.

\section{Forma de análise dos resultados}

Os resultados isotópicos foram submetidos à análise multivariada (MANOVA), e componentes principais quando houve diferença significativa, utilizando o pacote estatístico do software Minitab 16 (2010) para verificar a distinção entre os tratamentos.

\section{Resultados e Discussão}

Para os tecidos analisados de frangos de corte, a composição isotópica de $\delta^{13} \mathrm{Ce} \delta^{15} \mathrm{~N}$ foi influenciada pela extração lipídica ( $\mathrm{p}<0,01)$. Os tecidos desidratados apresentaram valores isotópicos de $\delta^{13} \mathrm{C}$ de $-19,87 \%$ o, $-19,79 \%$ e $-19,85 \%$, e de $\delta^{15} \mathrm{~N}$ de $1,76 \%, 2,00 \%$ e $3,19 \%$, respectivamente para músculo peitoral, coxa e fígado (Tabela 1). Posteriormente à extração da gordura por éter, os componentes de $\delta^{13} \mathrm{C}$ e $\delta^{15} \mathrm{~N}$ variaram $-19,52 \%$, $-19,51 \%$ o e $-19,37 \%$ o, e $1,75 \%$, $2,04 \%$ e $2,97 \%$, nessa ordem. Para a extração da gordura por clorofórmio/metanol, a variação de $\delta^{13} \mathrm{C}$ e $\delta^{15} \mathrm{~N}$ foi de $-19,70 \%,-19,66 \%$ e $-20,06 \%$, e $1,92 \%$, $2,17 \%$ e $3,33 \%$ para músculo peitoral, coxa e fígado, respectivamente. 
Contudo, para o fígado e músculo peitoral de frango, houve diferença dos componentes que sofreram extração lipídica por éter entre os componentes não desengordurados e os desengordurados por clorofórmio/metanol $(\mathrm{p}<0,01)$ (Figura 1). Para o músculo da coxa, entretanto, existiu diferença $(p<0,01)$ dos elementos que sofreram extração da gordura em clorofórmio/metanol aos elementos que sofreram extração da gordura por éter e os não desengordurados.

Quanto à diferenciação entre os tratamentos para o músculo peitoral, o primeiro componente (Tabela
2) sofreu maior influência dos valores isotópicos de nitrogênio, e o segundo sofreu maior influência dos valores isotópicos de carbono $(\mathrm{p}<0,01)$. No caso do músculo da coxa e fígado, o primeiro componente sofreu maior influência dos valores isotópicos de carbono e o segundo componente sofreu maior influência dos valores isotópicos $(\mathrm{p}<0,01)$.

A diferença isotópica causada pelos processos de extração lipídica foi menor que várias relatadas em literatura (Kiljunen et al., 2006; Sweeting et al., 2006; Post et al., 2007; Smyntek et al., 2007), porém similar à encontrada por Denadai et al. (2011).
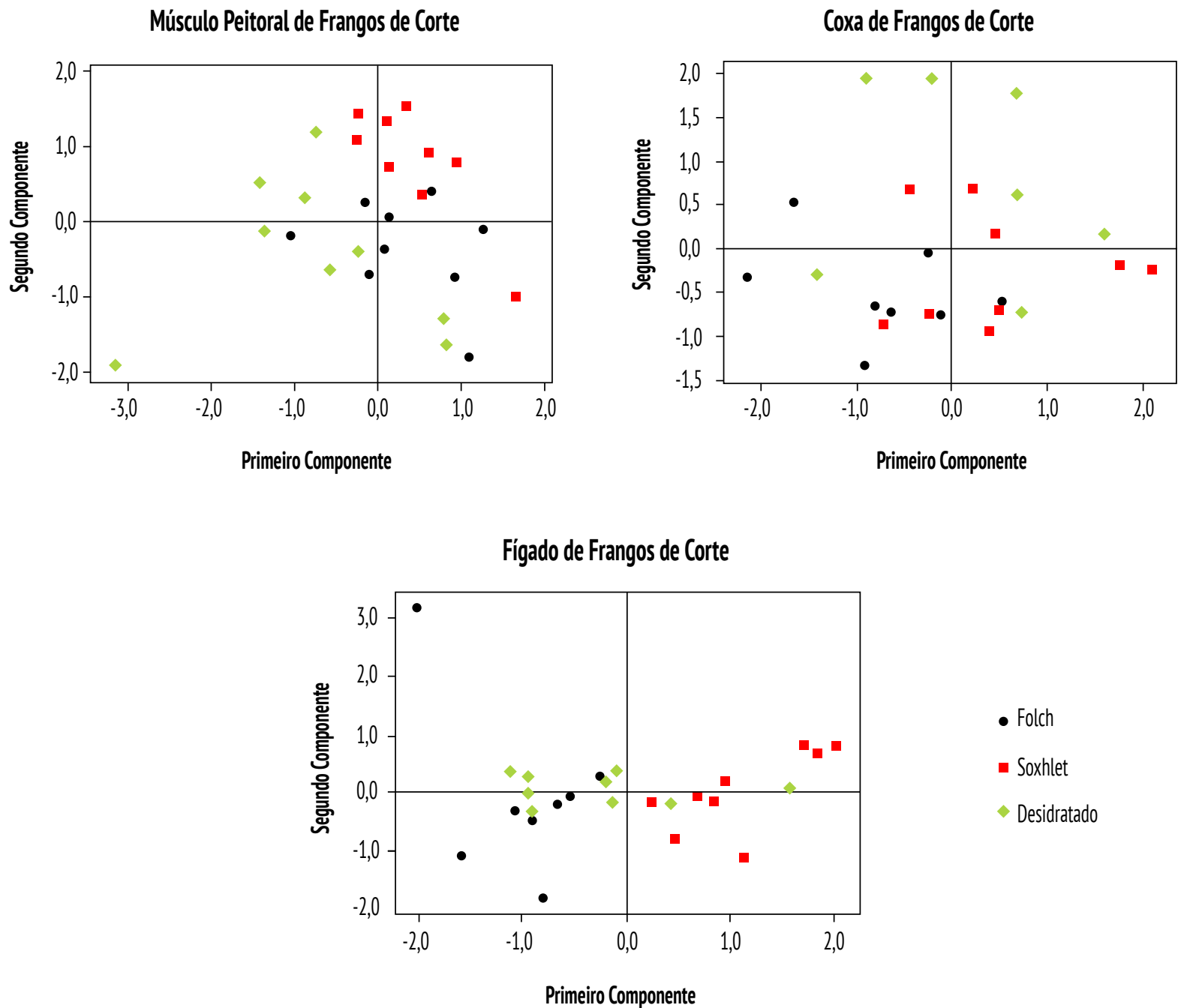

Figura 1 - Comparação entre os tratamentos com e sem extração lipídica em frangos de corte, a partir da análise de multivariância (MANOVA) do carbono-13 e nitrogênio-15. 
Tabela 1 - Valores isotópicos médios de tecidos de frangos de corte

\begin{tabular}{ccccc}
\hline & Isótopo & Desidratado & Soxhlet & Folch \\
\hline M.Peitoral $^{*}$ & $\delta^{13} \mathrm{C}$ & $-19,87 \pm 0,23$ & $-19,52 \pm 0,06$ & $-19,70 \pm 0,12$ \\
& $\delta^{15} \mathrm{~N}$ & $1,76 \pm 0,27$ & $1,75 \pm 0,21$ & $1,92 \pm 0,18$ \\
M.Coxa* $^{*}$ & $\delta^{13} \mathrm{C}$ & $-19,79 \pm 0,32$ & $-19,51 \pm 0,16$ & $-19,66 \pm 0,16$ \\
& $\delta^{15} \mathrm{~N}$ & $2,00 \pm 0,11$ & $2,04 \pm 0,08$ & $2,17 \pm 0,07$ \\
Fígado $^{*}$ & $\delta^{13} \mathrm{C}$ & $-19,85 \pm 0,32$ & $-19,37 \pm 0,18$ & $-20,06 \pm 0,63$ \\
& $\delta^{15} \mathrm{~N}$ & $3,19 \pm 0,14$ & $2,97 \pm 0,20$ & $3,33 \pm 0,21$ \\
\hline
\end{tabular}

Nota: * Par isotópico entre tratamentos diferente estatisticamente pela MANOVA $p<0,01 . \delta^{13} \mathrm{C}=$ carbono-13; $\delta^{15} \mathrm{~N}=$ nitrogênio-15.

Tabela 2 - Coeficiente de escores da análise de fatores (PC1 e PC2) para os tecidos de frangos de corte com e sem extração de gordura em função dos elementos analisados

\begin{tabular}{lrccccc}
\hline & \multicolumn{3}{c}{ Músculo Peitoral } & \multicolumn{2}{c}{ Músculo da coxa } & \multicolumn{2}{c}{ Fígado } \\
\hline Variável & PC1 & PC2 & PC1 & PC2 & PC1 & PC2 \\
Carbono* $^{*}$ & 0,096 & 0,995 & $-0,993$ & $-0,114$ & $-0,991$ & $-0,137$ \\
Nitrogênio* $^{*}$ & 0,995 & $-0,096$ & 0,114 & $-0,993$ & 0,137 & $-0,991$ \\
\hline
\end{tabular}

Nota: * Par isotópico entre tratamentos diferente estatisticamente pela multivariada discriminante $(\mathrm{p}<0,01)$.

Assim, para os tecidos analisados de suínos, a composição isotópica de $\delta^{13} \mathrm{C}$ e $\delta^{15} \mathrm{~N}$ dos tecidos foi influenciada pela extração lipídica $(\mathrm{p}<0,01)$. Os tecidos desidratados e não desengordurados exibiram valores isotópicos de $\delta^{13} \mathrm{C}$ de $-25,57 \%$, $-26,22 \%,-26,55 \%$ para músculo masseter, músculo semitendinoso e fígado, respectivamente, e de $\delta^{15} \mathrm{~N}$ de $4,44 \%$, 3,51\%o e 5,55\%o, respectivamente para músculo masseter, músculo semitendinoso e fígado (Tabela 3). Posteriormente à extração da gordura por éter, os componentes de $\delta^{13} \mathrm{C}$ e $\delta^{15} \mathrm{~N}$ variam $-24,93 \%$, $-24,38 \%$, $-26,72 \%$, e 4,69\%, 3,62\%o e 5,53\%o, nessa ordem para músculo masseter, músculo semitendinoso e fígado. Para a extração da gordura por clorofórmio/metanol, a variação de $\delta^{13} \mathrm{C}$ e $\delta^{15} \mathrm{~N}$ foi de $-25,51 \%$ o, $-24,91 \%$, $-27,40 \%$, e $4,61 \%$, 3,64\%o, 5,62\%o para músculo masseter, músculo semitendinoso e fígado (Tabela 3).

Para os músculos semitendinoso e masseter de suíno, porém, houve diferença dos componentes não desengordurados entre os componentes desengordurados em éter e os desengordurados por clorofómio/metanol (Figura 2). Para o fígado, verificou-se diferença entre os elementos que sofreram extração da gordura em éter e aqueles que sofreram extração da gordura por clorofórmio/ metanol e os não desengordurados. Quanto à diferenciação entre os tratamentos para fígado e músculos semitendinoso e masseter, o primeiro componente (Tabela 4) sofreu maior influência dos valores isotópicos de carbono, e o segundo componente dos valores isotópicos de nitrogênio $(\mathrm{p}<0,01)$.

Tabela 3 - Valores médios dos tecidos de suínos

\begin{tabular}{ccccc}
\hline & Isótopos & Desidratado & Soxhlet & Folch \\
\hline MM $^{*}$ & $\delta^{13} \mathrm{C}$ & $-25,57 \pm 0,56$ & $-24,93 \pm 0,93$ & $-25,51 \pm 0,77$ \\
& $\delta^{15} \mathrm{~N}$ & $4,44 \pm 0,26$ & $4,69 \pm 0,48$ & $4,61 \pm 0,20$ \\
MS $^{*}$ & $\delta^{13} \mathrm{C}$ & $-26,22 \pm 0,94$ & $-24,38 \pm 0,58$ & $-24,91 \pm 0,65$ \\
& $\delta^{15} \mathrm{~N}$ & $3,51 \pm 0,15$ & $3,62 \pm 0,19$ & $3,64 \pm 0,16$ \\
Fígado $^{*}$ & $\delta^{13} \mathrm{C}$ & $-26,55 \pm 2,75$ & $-26,72 \pm 0,27$ & $-27,40 \pm 0,34$ \\
& $\delta^{15} \mathrm{~N}$ & $5,55 \pm 0,20$ & $5,53 \pm 0,23$ & $5,62 \pm 0,23$ \\
\hline
\end{tabular}

Nota: * Par isotópico entre tratamentos diferentes estatisticamente pela MANOVA $(p<0,01) \cdot$ MM = músculo masseter; $M S$ = músculo semitendinoso. $\delta^{13} \mathrm{C}=$ carbono-13; $\delta^{15} \mathrm{~N}=$ nitrogênio-15.

Tabela 4 - Coeficiente de escores da análise de fatores (PC1 e PC2) para os tecidos de suínos com e sem extração de gordura em função dos elementos analisados

\begin{tabular}{lcccccc}
\hline & \multicolumn{2}{c}{ Masseter } & \multicolumn{2}{c}{ Semitendinoso } & \multicolumn{2}{c}{ Fígado } \\
\hline Variável & $\mathrm{PC1}^{*}$ & $\mathrm{PC2}$ & $\mathrm{PC1}$ & $\mathrm{PC2}$ & $\mathrm{PC1}$ & $\mathrm{PC2}$ \\
Carbono & $-0,974$ & $-0,226$ & $-1,000$ & $-0,014$ & $-0,975$ & $-0,223$ \\
Nitrogênio & 0,226 & $-0,974$ & 0,014 & $-1,000$ & 0,223 & $-0,975$ \\
\hline
\end{tabular}

Nota: *Par isotópico entre tratamentos diferente estatisticamente pela multivariada discriminante $(p<0,01)$.

A diferença isotópica causada pelos processos de extração lipídica por éter foi menor que o valor encontrado por Lo Tierzo (2012) para o músculo masseter.

A composição isotópica de $\delta^{13} \mathrm{C}$ e $\delta^{15} \mathrm{~N}$ do filé de pintado foi influenciada pela extração lipídica 
(p < 0,01). À extração da gordura por éter, os componentes de $\delta^{13} \mathrm{C}$ e $\delta^{15} \mathrm{~N}$ variaram -20,21\%o e $7,81 \%$, respectivamente (Tabela 5). 0 tecido que não sofreu extração lipídica mostrou valor isotópico de $\delta^{13} \mathrm{C}$ de $-20,65 \%$ e de $\delta^{15} \mathrm{~N}$ de $7,77 \%$ o (Tabela 5). Em seguida, a extração da gordura por clorofórmio/ metanol mostrou variação de $\delta^{13} \mathrm{Ce} \delta^{15} \mathrm{~N}$ de $-20,53 \%$ e 7,74\%, nessa ordem, para o filé (Tabela 5).

Houve, entretanto, diferença entre os componentes desengordurados por éter e os que não foram desengordurados por clorofórmio/metanol do filé de pintado (Figura 3).
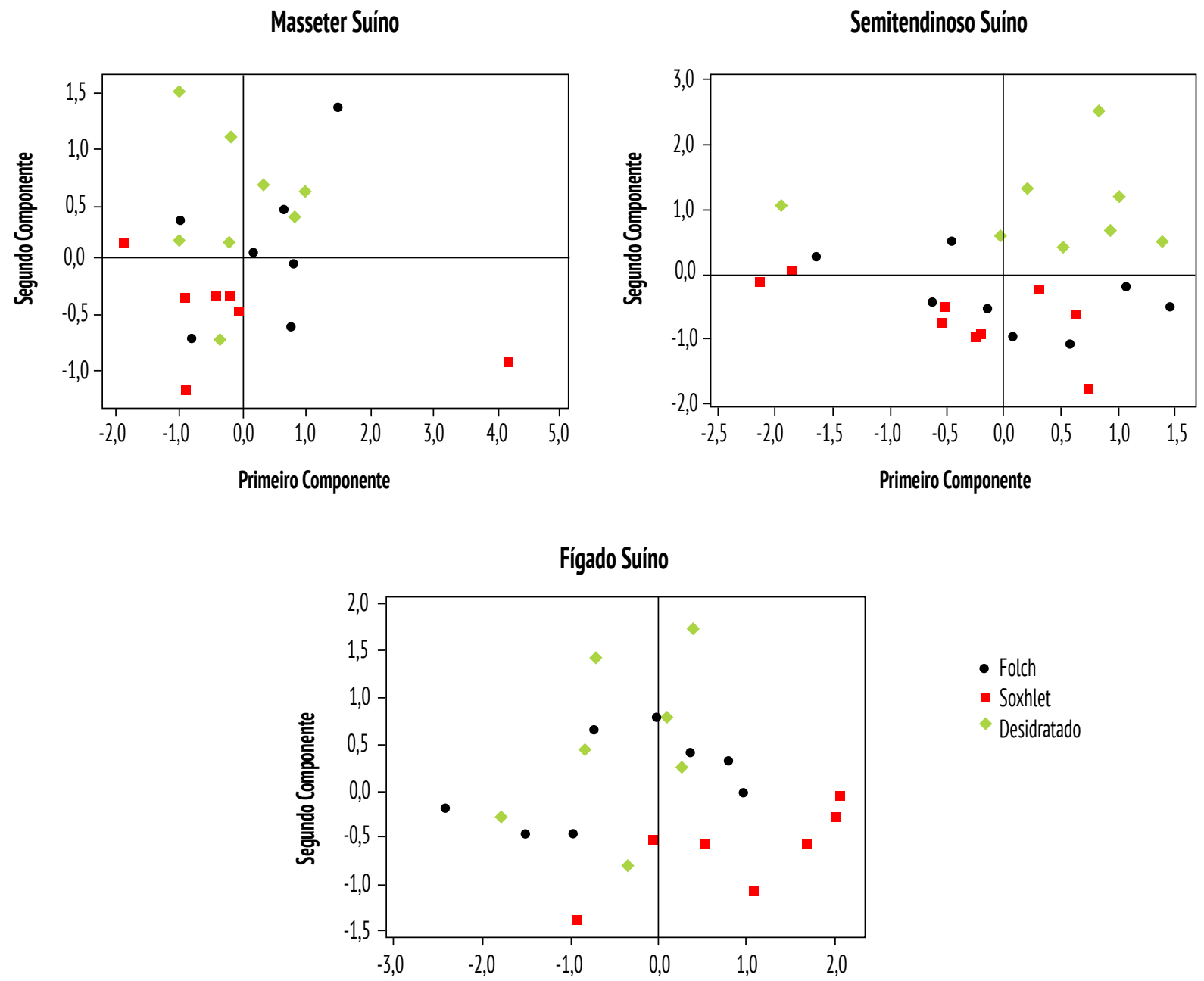

Primeiro Componente

Figura 2 - Comparação entre os tratamentos com e sem extração lipídica em suínos, a partir da análise de multivariância (MANOVA) do carbono-13 e nitrogênio-15.

Quanto à diferenciação entre os tratamentos para o filé de pintado, o primeiro componente (Tabela 6) sofreu maior influência dos valores isotópicos de carbono, e o segundo componente sofreu maior influênciados dos valores isotópicos de nitrogênio.
Já para o filé de tilápia, a composição isotópica de $\delta^{13} \mathrm{C}$ e de $\delta^{15} \mathrm{~N}$ do tecido não sofreu influência pela extração lipídica. 0 tecido não desengordurado exibiu valor isotópico de $\delta^{13} \mathrm{C}$ de $-23,02 \%$ e de $\delta^{15} \mathrm{~N}$ de 7,70\%o (Tabela 5). Posteriormente à extração 
da gordura por éter, o componente de $\delta^{13} \mathrm{C}$ variou $-22,60 \%$ e o de $\delta^{15} \mathrm{~N}$ variou $7,70 \%$ o (Tabela 5). Quanto à extração lipídica por clorofórmio/ metanol, a variante de $\delta^{13} \mathrm{C}$ e $\delta^{15} \mathrm{~N}$ foi de $-22,83 \%$ e $7,71 \%$, respectivamente, para o filé analisado.

Tabela 5 - Valores isotópicos médios dos tecidos de peixes

\begin{tabular}{ccccc}
\hline & Isótopos & Desidratado & Soxhlet & Folch \\
\hline Pintado* $^{*}$ & $\delta^{13} \mathrm{C}$ & $-20,65 \pm 0,14$ & $-20,21 \pm 0,14$ & $-20,53 \pm 0,14$ \\
& $\delta^{15} \mathrm{~N}$ & $7,77 \pm 0,19$ & $7,81 \pm 0,20$ & $7,74 \pm 0,39$ \\
Tilápia $^{*}$ & $\delta^{13} \mathrm{C}$ & $-23,02 \pm 1,89$ & $-22,60 \pm 1,82$ & $-22,83 \pm 1,96$ \\
& $\delta^{15} \mathrm{~N}$ & $7,70 \pm 0,32$ & $7,70 \pm 0,32$ & $7,71 \pm 0,34$ \\
\hline
\end{tabular}

Nota: *Par isotópico entre tratamentos diferente estatisticamente pela MANOVA $(p<0,01) \cdot \delta^{13} \mathrm{C}=$ carbono- $13 ; \delta^{15} \mathrm{~N}=$ nitrogênio-15.

Tabela 6 - Coeficiente de escores da análise de fatores para o pintado com e sem extração de gordura em função dos elementos analisados

\begin{tabular}{ccc}
\hline & Filé de Pintado \\
\hline Variável & PC1 & PC2 \\
Carbono* $^{*}$ & 0,993 & $-0,121$ \\
Nitrogênio* $^{*}$ & 0,121 & 0,993 \\
\hline
\end{tabular}

Nota: *Par isotópico entre tratamentos diferente estatisticamente pela multivariada discriminante $p<0,01$.

Não houve diferenciação dos valores isotópicos médios entre os diferentes métodos de extração lipídica (Figura 4).

A composição isotópica de $\delta^{13} \mathrm{C}$ e de $\delta^{15} \mathrm{~N}$ dos tecidos de bovinos não apresentou influência pela extração lipídica. Os tecidos não desengordurados exibiram valores isotópicos de $\delta^{13} \mathrm{C}$ e de $\delta^{15} \mathrm{~N}$ de $-16,78 \%$, $-15,65 \%$ o, $-18,54 \%$, e $7,07 \%$, 5,99\%o e 7,49\%o, respectivamente para acém, lagarto e fígado. Posteriormente à extração da gordura por éter, mostraram valores isotópicos de $\delta^{13} \mathrm{C}$ de $-16,40 \%$, $-15,46 \%$, $-18,38 \%$, e de $\delta^{15} \mathrm{~N}$ de $7,15 \%$, $6,11 \%$ e 6,68\%o, nessa ordem para acém, lagarto e fígado. Em extração lipídica por clorofórmio/ metanol, a variação de $\delta^{13} \mathrm{C}$ e $\delta^{15} \mathrm{~N}$ foi de $-16,36 \%$, $-14,05 \%$, $-18,70 \%$, e $7,26 \%, 6,15 \%$ e $7,48 \%$, respectivamente (Tabela 7).
Filé de Pintado

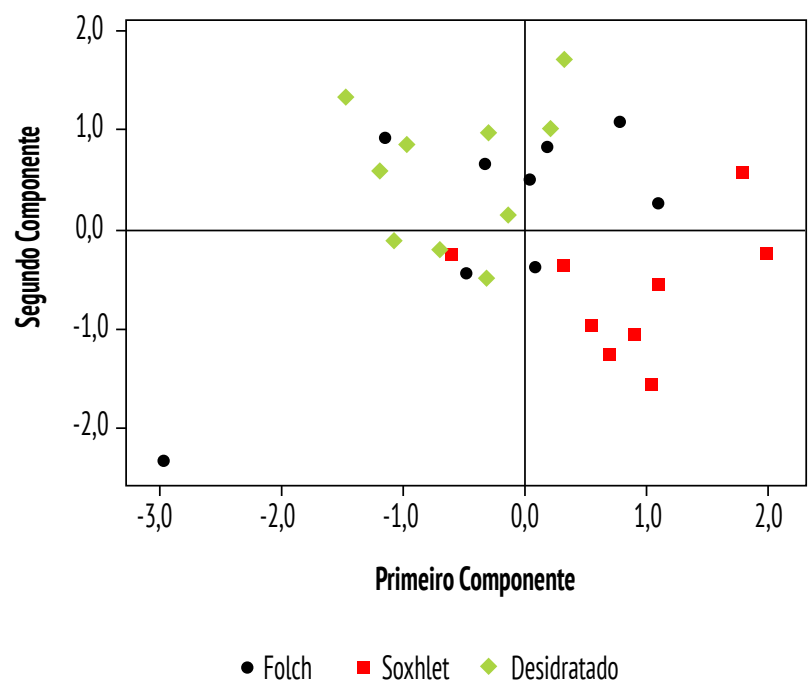

Figura 3 - Comparação entre os tratamentos com e sem extração lipídica em filé de pintado, a partir da análise de multivariância (MANOVA) do carbono-13 e nitrogênio-15.

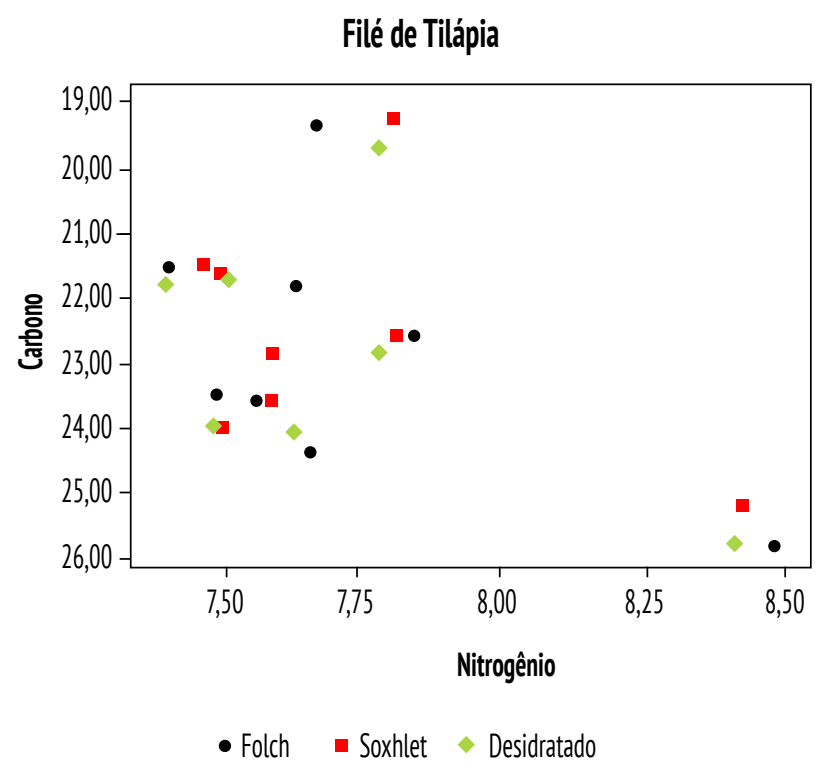

Figura 4 - Valores isotópicos de carbono-13 e nitrogênio-15 em filé de tilápia submetidos a diferentes processos de extração lipídica.

Não houve diferença entre os elementos não desengordurados e os desengordurados por clorofórmio/metanol e éter (Figura 5). 

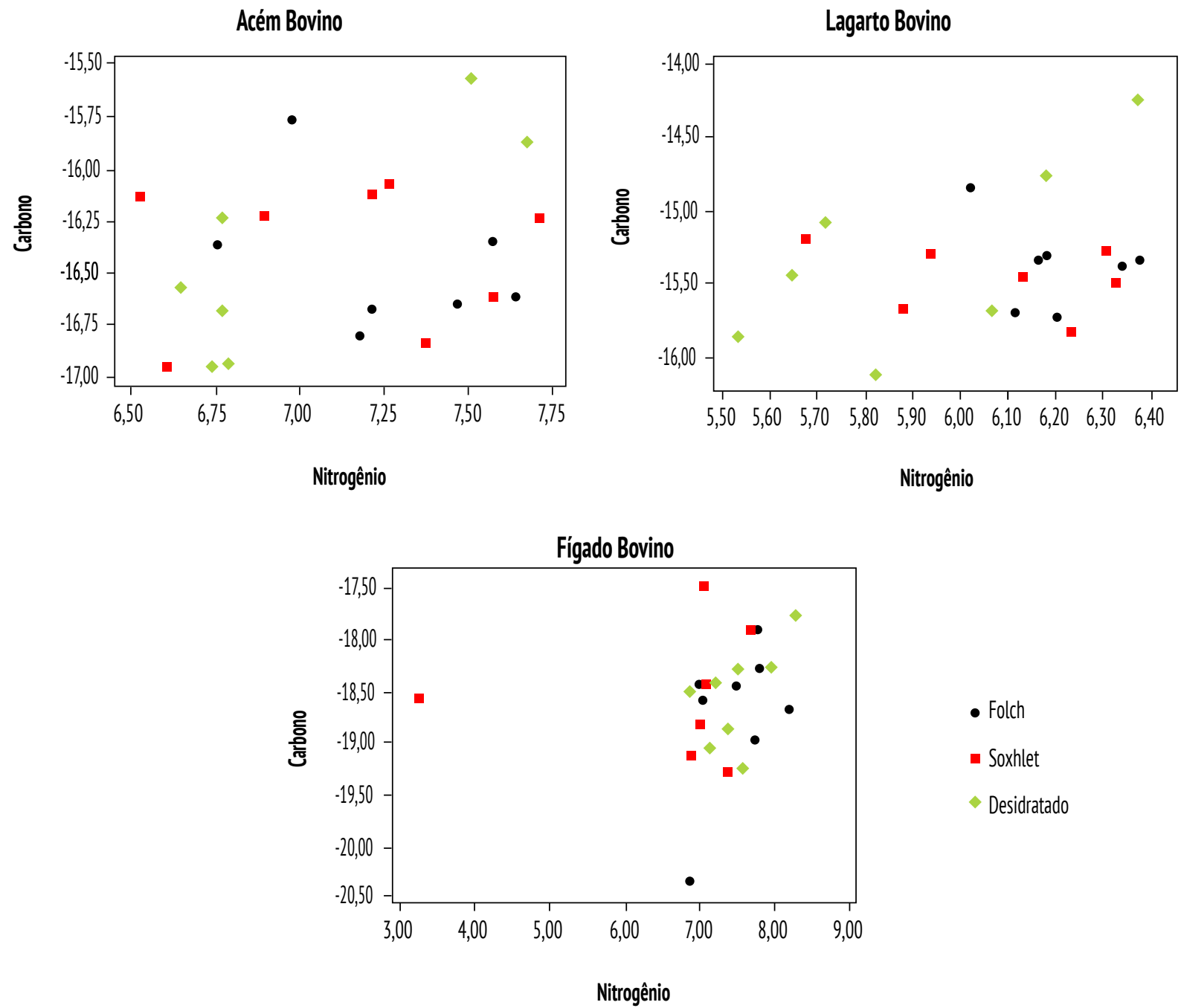

Figura 5 - Valores isotópicos de carbono-13 e nitrogênio-15 em tecidos de bovinos submetidos a diferentes processos de extração lipídica.

Tabela 7 - Valores isotópicos médios dos tecidos de bovinos de corte

\begin{tabular}{ccccc}
\hline & Isótopos & Desidratado & Soxhlet & Folch \\
\hline Acém ${ }^{*}$ & $\delta^{13} \mathrm{C}$ & $-16,78 \pm 1,17$ & $-16,40 \pm 0,34$ & $-16,36 \pm 0,43$ \\
& $\delta^{15} \mathrm{~N}$ & $7,07 \pm 0,46$ & $7,15 \pm 0,43$ & $7,26 \pm 0,32$ \\
Lagarto $^{*}$ & $\delta^{13} \mathrm{C}$ & $-15,65 \pm 1,11$ & $-15,46 \pm 0,21$ & $-14,05 \pm 3,55$ \\
& $\delta^{15} \mathrm{~N}$ & $5,99 \pm 0,38$ & $6,11 \pm 0,26$ & $6,15 \pm 0,19$ \\
Fígado* $^{*}$ & $\delta^{13} \mathrm{C}$ & $-18,54 \pm 0,49$ & $-18,38 \pm 0,70$ & $-18,70 \pm 0,73$ \\
& $\delta^{15} \mathrm{~N}$ & $7,49 \pm 0,46$ & $6,68 \pm 1,40$ & $7,48 \pm 0,47$ \\
\hline
\end{tabular}

Nota: *Par isotópico entre tratamentos diferente estatisticamente pela MANOVA $(p<0,01) \cdot \delta^{13} \mathrm{C}=$ carbono-13; $\delta^{15} \mathrm{~N}=$ nitrogênio- 15 .
Aceitando que o conteúdo lipídico dentro do tecido muscular das espécies pode ser altamente variável, dependendo de fatores como tamanho, sexo, condição de maturidade e época de amostragem, a extração de lipídios, para determinação isotópica, é considerada como prática corrente para remover os carbonos empobrecidos dos lipídios. Isto permite uma padronização ao examinar diferentes tecidos de várias espécies (Hussey et al. 2008).

Essa correção aritmética de lipídios tem sido desenvolvida para tecidos de peixes e invertebrados (Kiljunen et al., 2006; Sweeting et al., 2006; Smyntek 
et al., 2007), no entanto, a confiabilidade destas correções para gema de ovo, com o seu elevado teor lipídico, não foi estabelecida (Post et al., 2007; Logan et al., 2008; Mintenbeck, et al., 2008). A atual falta de correção aritmética confiável faz a extração química dos lipídios necessária para a análise de isótopos estáveis de carbono na gema (Gauthier et al., 2003; Hobson et al., 2005; Bond et al., 2007).

Assim, as diferenças no conteúdo lipídico de amostras podem confundir a interpretação dos isótopos estáveis de carbono (Post et al., 2007), e muitos estudos ecológicos removem quimicamente os lipídios dos tecidos ou aplicam uma correção aritmética para explicar a maior abundância de isótopos de carbono mais leves em lipídios (Kiljunen et al., 2006; Sweeting et al., 2006; Post et al., 2007).

Este estudo, com diversos tecidos de espécies com finalidade zootécnica, levanta, porém, a hipótese da não realização da extração lipídica, pois a variação isotópica nos tecidos estudados foi insignificativa quando comparados aos estudos ecológicos. Em estudos ecológicos, a variação encontrada é em torno de 3\%o para carbono-13 e de 5\%o para nitrogenio-15 (Kennedy e Krouse, 1990). Essa maior variação em espécies da natureza pode ser causada por uma série de fatores, tais como dieta não constante dos animais, dieta heterogênea durante o dia e deposição lipídica desses animais, que deve possuir finalidade distinta das espécies domésticas, ou seja, para controle de temperatura, armazenamento de energia para voos migratórios, dentre outros (Kennedy e Krouse, 1990; Hobson e Clark, 1992).

\section{Conclusão}

A extração lipídica por éter interferiu nos valores isotópicos dos tecidos do peito e fígado de frangos de corte, fígado de suíno e filé de pintado. A extração lipídica por clorofórmio/metanol interferiu nos valores isotópicos dos tecidos do músculo da coxa de frangos de corte, músculo masseter e semitendinoso de suínos. Entretanto não ocorreram alterações nos valores isotópicos, independentemente do método de extração para os tecidos de bovino e filé de tilápia. Sugerese, portanto, a não extração lipídica em tecidos de animais com finalidade zootécnica, a fim de diminuir a interferência dos processos de extração lipídica nos valores isotópicos dos tecidos animais.

\section{Referências}

Bond JC, Esler D, Hobson KA. Isotopic evidence for sources of nutrients allocated to clutch formation by Harlequin Ducks. Condor. 2007;109(3):698-704.

Bricout J, Koziet J. Control of the authenticity of orange juice by isotopic analysis. J. Agric. Food Chem. 1987;35(5):758-60.

Brookes ST, Barrie A, Davies JE. A rapid 13C/12C test for determination of corn-syrups in honey. J Assoc Off Anal Chem. 1991;74(4):627-9.

Bury S, Pinkerton M, Thompson D, Cherel Y, Brown J. The effect of lipid-extraction onhigh lipidcontent Notothenioid (anti-freeze) fish from the Ross sea, Antarctica. 6th International Conference on Applications of Stable Isotope Techniques to Ecological Studies; 25-29 ago 2008; Honolulu, EUA. Honolulu: University of Hawai'i at Mānoa; 2008.

Carrijo AS, Pezzato AC, Ducatti C. Avaliação do metabolismo nutricional em poedeiraspela técnica dos isótopos estáveis do carbono $\left({ }^{13} \mathrm{C} /{ }^{12} \mathrm{C}\right)$. Rev Bras Cienc Avic. 2000;2(3):209-18.

Denadai JC, Ducatti C, Sartori JR, Madeira LA, Pizzolante CC, Sartori MMP. Rastreabilidade em ovos por isótopos estáveis. XII Congresso Latino-Americano de Avicultura; 6-9 set 2011; Buenos Aires, Argentina.

Denadai JC, Ducatti C, Sartori JR, Pezzato AC, Móri C, Gottmann R, et al. The traceability of animal meals in layer diets as detected by stable carbon and nitrogen isotope analyses of eggs. Rev Bras Cienc Avic. 2008;10(3):189-94

Folch J, Lees M, Stanley GHS. A simple method for the isolation and purification of total lipids from animal tissues. J Biol Chem. 1957;226(1):497-509.

Gauthier G, Bêty J, Hobson KA. Are Greater Snow Geese capital breeders? New evidence from a stable-isotope model. Ecology. 2003;84(12):3250-64. 
González-Martin I, González-Pérez C, Hernández-Méndez J, Marqués-Macias E, Sanz Poveda F. Use of isotope analysis to characterize meat from Iberian-breed swine. Meat Sci. 1999;52(4):437-41.

González-Martin I, González-Pérez C, HernándezMéndez J, Sánchez González C. Differentiation of dietary regimen of Iberian swine by means of isotopic analysis of carbon and sulphur in hepatic tissue. Meat Sci. 2001;58(1):25-30.

Hargin KD. Authenticity issues in meat and meat products. Meat Sci. 1996;43(Supl 1):S277-89.

Hobson KA, Clark RG. Assessing avian diets using stable isotopes I: turnover of $13 \mathrm{C}$ in tissues. Condor. 1992;94(1):181-8.

Hobson KA, Thompson JE, Evans MR, Boyd S. Tracing nutrient allocation to reproduction in Barrow's Goldeneye. J Wildl Manage. 2005;69(3):1221-8.

Hussey NE, McCarthy ID, Dudley SFJ, Fisk AT. Sharks vs. The rest of the world: lipid extraction and stable isotopes. 6th International Conference on Applications of Stable Isotope Techniques to Ecological Studies; 25-29 ago 2008; Honolulu, EUA. Honolulu: University of Hawai'i at Mānoa; 2008.

Kennedy BV, Krouse HR. Isotope fractionation by plants and animals: implications for nutrition research. Can J Physiol Pharmacol. 1990;68(7):960-72.

Kiljunen M, Grey J, Sinisalo T, Harrod C, Immonen H, Jones RI. A revised model for lipid-normalizing $\delta 13 \mathrm{C}$ values from aquatic organisms, with implications for isotopemixing models. J Appl Ecol. 2006;43(6):1213-22.

Koziet], Rossmann A, Martin GJ, Ashurst PR. Determination of carbon-13 content of sugars of fruit and vegetable juices: A European inter-laboratory comparison. Anal Chim Acta. 1993;271(1):31-8.

Lo Tierzo V. Turnover do carbono nos tecidos de suínos em diferentes fases de crescimento [tese]. Botucatu: Universidade Estadual Paulista; 2012. 52 p.
Logan JM, Jardine TD, Miller TJ, Bunn SE, Cunjak RA, Lutcavage ME. Lipid corrections in carbon and nitrogen stable isotope analyses: Comparison of chemical extraction and modelling methods. J Anim Ecol. 2008;77(4):838-46.

Manca G, Camin F, Coloru G, Del Caro A, Detentori D, Franco MA, et al. Characterisation of the geographical origin of Pecorino Sardo cheese by casein stable isotope $\left({ }^{13} \mathrm{C} /{ }^{12} \mathrm{C}\right.$ and $\left.{ }^{15} \mathrm{~N} /{ }^{14} \mathrm{~N}\right)$ ratios and free amino acid ratios. J Agric Food Chem. 200;49(3):1404-9.

Martin GJ, Guillou C, Martin ML, Cabanis MT, Tep Y, Aerny J. Natural factors of isotope fractionation and the characterization of wines. J. Agric. Food Chem. 1988;36(2):316-22.

Minitab Statistical Software, version 16. State College, PA: Minitab Inc; 2010.

Mintenbeck K, Brey T, Jacob U, Knust R, Struck U. How to account for the lipid effect on carbon stable-isotope ratio ( $\delta 13 \mathrm{C})$ : Sample treatment effects and model bias. J Fish Biol. 2008;72(4):815-30.

Monin G. Recent methods for predicting quality of whole meat. Meat Sci. 1998;49(Supl 1):S231-43.

Oliveira RP. Rastreabilidade da farinha de vísceras de aves na alimentação de frangos de corte pela técnica dos isótopos estáveis ( $\delta 13 \mathrm{C}$ e $\delta 15 \mathrm{~N})$ [tese]. Botucatu: Universidade Estadual Paulista; 2005. 109 p.

Piasentier E, Valusso R, Camin F, Versini G. Stable isotope ratio analysis for authentication of lamb meat. Meat Sci. 2003;64(3):239-47.

Post DM, Layman CA, Arrington DA, Takimoto G, Quattrochi J, Montaña CG. Getting to the fat of the matter: Models, methods and assumptions for dealing with lipids in stable isotope analyses. Oecologia. 2007;152(1):179-89.

Rossmann A, Haberhauer G, Hölzl S, Horn P, Pichlmayer F, Voerkelius $S$. The potential of multielement stable isotope analysis for regional origin assignment of butter. Eur Food Res Technol. 2000;211(1):32-40. 
SmyntekPM, Teece MA,Schulz KL, Thackeray SJ. Astandard protocol for stable isotope analysis of zooplankton in aquatic food web research using mass balance correction models. Limnol Oceanogr. 2007;52(5):2135-46.

Sweeting CJ, Polunin NV, Jennings S. Effects of chemical lipid extraction and arithmetic lipid correction on stable isotope ratios of fish tissues. Rapid Commun Mass Spectrom. 2006;20(4):595-601.

White JW, Winters K, Martin P, Rossmann A. Stable carbon isotope ratio analysis of honey: validation of internal standard procedure for worldwide application. J Assoc Off Anal Chem. 1998;81(3):610-9. 